\title{
Coordinated UAV Standoff Tracking of Moving Target based on Lyapunov Vector Fields
}

\author{
Tagir Z. Muslimov \\ Ufa State Aviation Technical University \\ Ufa, Russia \\ tagir.muslimov@gmail.com
}

\author{
Rustem A. Munasypov \\ Ufa State Aviation Technical University \\ Ufa, Russia \\ rust40@mail.ru
}

\begin{abstract}
This paper proposes an approach to cooperative tracking of a moving target using a system of multiple fixed-wing UAVs. In this scenario, the task is to coordinate a UAV group so that it could follow a moving circular path while keeping specified (and not necessarily identical) spacing. Many papers dwell upon path following problems for UAV formations; solutions they propose include Lyapunov vector fields. This article particularly uses a different method that revolves around a decentralized guidance Lyapunov vector field for path following, which field is non-uniform in both direction and magnitude. The advantage of proposed strategy lies in the global asymptotic stability it provides, which helps not only create a UAV formation around the target faster, but also keep it stable shall the formation fail to precisely follow its specified orbit. The authors hereof analyzed the consensus-based coordination architecture in the form of a decentralized open chain. For space considerations, this paper only describes a target moving at a constant speed. MATLAB/Simulink modeling based on complete non-linear flying-wing UAV models shows that the proposed approach efficient.
\end{abstract}

Keywords-distributed control, swarm behavior, cooperative control, UAV flocking, UAV consensus, guidance vector field, multiUAV system

\section{INTRODUCTION}

Recent years have seen multifaceted research into control of unmanned aerial vehicle (UAV) groups. Focus is on planning the movements of UAV groups [1], on the one hand, and on trajectory control on the other hand [2]. However, controlling an autonomous swarm formation, including cooperative path following, is a problem yet to be solved. Decentralized approaches seem promising and advantageous when it comes to controlling a formation. Researchers in the field are often inspired by swarm behavior as seen in the wild [3].

Tracking a moving ground target is a baseline UAV scenario [4]. For fixed-wing UAVs, this can be complicated, as these aircraft have to move continually at some minimum speed and cannot hover. A UAV group can estimate the target's movement more accurately and might generally be more reliable [5]. In this case, a group must not only keep a specific distance to the target, but also attain and maintain specified phase shift angles between the UAVs in flight.

Various strategies for cooperative target tracking have been proposed. Lyapunov guidance vector fields are an efficient method therefor [6]. This approach has been furthered in [7]-

The reported study was funded by RFBR according to the research project № 18-08-01299.
[9], where it helped attain circular formations that had specified phase shift angles between the UAVs. Unlike this paper, those above were about attaining a formation of UAVs that were assumed to have already been set on a circular path. This assumption might be impractical, as, for instance, wind shifts might cause an UAV to "derail".

Paper [10] uses a vector field non-uniform in direction only, i.e. UAVs maintain constant flight speeds. Article [11], unlike this one, ignores the input saturations present in the real-world autopilot-UAV systems; besides, the formation is equidistant at the arc and is not an arbitrary pre-specified formation. Paper [12] dwells upon a system of models that simulate unicycles moving at constant speeds; it, too, ignores input saturations.

This article proposes using Lyapunov vector fields that are non-uniform in both direction and magnitude [13]. Thus, the cooperative UAV speed controller will be engaged as soon as the formation begins to take shape. The control laws themselves are adjusted for input saturations.

In general, this approach can be considered an instance of cooperative path following, where the path is moving. Similar approaches have been well-covered in literature in application to single UAVs and mobile robots [14], [15]. However, it becomes a far more complex task when it has to be coupled with attaining a formation and stabilizing it at the same time.

\section{UAV MODEL AND PRELIMINARY REMARKS}

For space considerations, assume each UAV has data on the target speed and direction. If the target movement parameters are not known in advance, adaptive estimation algorithms could be of use.

\section{A. Topology of UAV Group Coordination}

The topology of coordinating UAVs as autonomous agents in a group follows a typical graph-based description, e.g. one presented in [16]. This study uses "open-chain" consensus-based coordination similar to that in [13], [17], [18]. Thus, each UAV coordinates with the two extreme UAVs and the two adjacent ones. An extreme UAV only has one adjacent counterpart.

The control action vector can thus be calculated similarly to [13]. This vector can be found as proposed in [19], i.e. derived from some elements of the vector of all possible relative phaseshift angle errors $\overline{\mathbf{e}}_{\theta}=\left(e_{i, j}\right) \in \mathbb{R}^{N(N-1) \times 1}$, where $\mathbb{R}^{N(N-1) \times 1}-$ is the space of matrices sized $N(N-1) \times 1$ with elements from $\mathbb{R}$, 
$e_{i, j}-$ is the error for the immediately coordinating agents: $i$ th and $j$ th. Which elements should be selected depends on the architecture of inter-agent coordination; for this paper, the control action vector is as follows:

$$
\mathbf{e}_{\theta}=\left[\begin{array}{c}
e_{1} \\
\vdots \\
e_{k} \\
\vdots \\
e_{N}
\end{array}\right]=\left[\begin{array}{c}
\hat{e}_{12} \\
\vdots \\
-\hat{e}_{k-1, k}+\hat{e}_{k, k+1} \\
\vdots \\
-\hat{e}_{N-1, N}
\end{array}\right]=\hat{\mathbf{M}}_{\theta} \breve{\mathbf{e}}_{\theta}+\mathbf{D},
$$

where $\mathbf{D}=-\mathbf{M}_{\theta} \mathbf{H}_{\theta}^{-1}\left(\mathbf{P}_{\theta d}^{\mathrm{T}}, \hat{P}_{\theta}\right)^{\mathrm{T}}-$ is the system control vector in the space of relative states (a space of $(N-1)$ dimensions spanned by the columns of the coordination graph incidence matrix [20]), $\mathbf{H}_{\theta}-$ is the matrix that specifies the agents to set the relative spacing between, which matrix is defined as follows:

$$
\mathbf{H}_{\theta}=\left[\begin{array}{c}
\mathbf{q}_{1} \\
\mathbf{q}_{2} \\
\vdots \\
\mathbf{q}_{N}
\end{array}\right], \quad \mathbf{q}_{i}=\left[\begin{array}{c}
\vdots \\
1 \\
\vdots \\
-1 \\
\vdots
\end{array}\right]^{\mathrm{T}}, i<N, \mathbf{q}_{N}=\left[\begin{array}{c}
1 \\
1 \\
\vdots \\
1
\end{array}\right]^{\mathrm{T}},
$$

whereby $\mathbf{H}_{\theta} \in \mathbb{R}^{N \times N}, \mathbf{q}_{i} \in \mathbb{R}^{1 \times N}$, the positions of «1» и «-1» in $\mathbf{q}_{i}$ being determined according to the coordination graph structure;

$\mathbf{P}_{\theta d} \in \mathbb{R}^{(N-1) \times 1}-$ is the vector of desired inter-UAV phase shift angles, $\hat{P}_{\theta}=\sum_{k=1}^{N} \varphi_{k}-$ is the total of the current UAV phase angles in the inertial co-ordinate system;

$\breve{\mathbf{e}}_{\theta}=\left(\breve{e}_{i, i+1}\right)_{i=1}^{N-1} \in \mathbb{R}^{(N-1) \times 1}-$ is the vector of current phase shift angles for immediately co-ordinating agents calculated by scalar triple product, e.g. when for ultimate clockwise movement:

if $\mathbf{n} \cdot\left(\mathbf{d}_{i} \times \mathbf{d}_{i+1}\right) \geq 0$, then $\breve{e}_{i, i+1}=\beta=\arccos \frac{\left(\mathbf{d}_{i}, \mathbf{d}_{i+1}\right)}{\left\|\mathbf{d}_{i}\right\|\left\|\mathbf{d}_{i+1}\right\|}$, else $\breve{e}_{i, i+1}=2 \pi-\beta$, where $\mathbf{d}_{k}, k \in 1,2, \ldots, N-$ is the vector of distance from the movig target to the aircraft at the current time, $\mathbf{n}=(0,0,1)^{\mathrm{T}}$;

$\mathbf{M}_{\theta} \in \mathbb{R}^{N \times N}$ - is the coordination matrix, which in case of decentralized neighbor-neighbor coordination used herein is as follows:

$$
\mathbf{M}_{\theta}=\left[\begin{array}{ccccc}
-1 & 1 & 0 & \cdots & 0 \\
1 & -2 & \ddots & \ddots & \vdots \\
0 & \ddots & \ddots & 1 & 0 \\
\vdots & \ddots & 1 & -2 & 1 \\
0 & \cdots & 0 & 1 & -1
\end{array}\right]
$$

$\hat{\mathbf{M}}_{\theta} \in \mathbb{R}^{N \times(N-1)}-$ is the matrix derived from $\mathbf{M}_{\theta} \mathbf{H}_{\theta}^{-1}$ by deleting the $N$ th column.

\section{B. UAV Model}

For space considerations, assume each UAV has been commanded to maintain a constant flight altitude. Thus, the formation will take shape in a horizontal-plane projection.

For numerical simulation, use a complete nonlinear UAV dynamics model. If the autopilot functions as supposed, the high-level UAV model can be rendered as a second-order unicycle model as in [13]:

$$
\dot{x}=v^{c} \sin \chi, \dot{y}=v^{c} \cos \chi, \dot{\chi}=a_{\chi}\left(\chi^{c}-\chi\right),
$$

where $x$ is the UAV coordinate along the eastward axis in the inertial coordinate system, $y$ is the UAV coordinate along the northward axis in the inertial coordinate system, $\chi$ is the current heading angle, $\chi^{c}$ is the heading angle loop input, $v^{c}$ is the groundspeed loop input, $a_{\chi}$ is a positive constant determined by the autopilot settings and UAV specifications.

For the model (2), generate a globally asymptotically stable guidance vector field, following which is effectively cooperative path following, where the path is moving.

When synthesizing the control laws, the following input constraints should be born in mind:

$$
U \triangleq\left\{v^{c}, \dot{\chi}^{c} \mid 0<v_{\min } \leq v^{c} \leq v_{\max },-\dot{\chi}_{\max } \leq \dot{\chi}^{c} \leq \dot{\chi}_{\max }\right\} .
$$

The circular path a UAV formation must follow when flying around the target can be specified in terms of the center $\mathbf{c}=\left(c_{e}(t), c_{n}(t), h(t)\right) \in \mathbb{R}^{3}$, radius $\rho \in \mathbb{R}$, and direction of rotation $\lambda \in\{-1,1\}[21]$

$$
P_{o} \triangleq\left\{\mathbf{r} \in \mathbb{R}^{3}: \mathbf{r}=\mathbf{c}+\lambda \rho\left(\cos \varphi_{i}, \sin \varphi_{i}, 0\right)^{\mathrm{T}}, \varphi \in[0,2 \pi)\right\},
$$

where $c_{e}$ is center-of-circle coordinates along the eastward axis in the inertial coordinate system; $c_{n}$ is the center-of-circle coordinates along the northward axis in the inertial coordinate system; $h$ is the center-of-circle altitude, MASL; $\lambda=1$ defines the clockwise movement, while $\lambda=-1$ defines the counterclockwise movement; $\varphi_{i}$ is the current phase angle of the $i$ th UAV.

Unlike in [13], the center-of-circle coordinates here are not constant and may change over time, as they are also the target coordinates.

\section{Control Objectives}

A UAV group that uses consensus-based coordination must follow a moving path (4) while attaining and maintaining a formation. The phase shift angles can be arbitrarily set in advance rather than limited to being equidistant. For simplicity, consider a target moving at a constant speed and following a rectilinear trajectory. 


\section{VECTOR FIELD FOR TARGET TRACKING (NON-UNIFORM IN MAGNITUDE AND DIRECTION)}

Due to the significant oscillation observed when a UAV group is tracking a target, the speed and heading angle control laws from [13] have been modified to add derivative signals. Since direct differentiation will increase noise, these signals can be calculated analytically by measuring and exchanging positioning data between UAVs. Thus, the UAV speed control law is written as follows:

$$
\mathbf{v}^{c}=\left[\begin{array}{llll}
v_{1} & v_{2} & \ldots & v_{N}
\end{array}\right]^{\mathrm{T}}=v \mathbf{1}_{N}+\mathbf{L},
$$

where $\quad \mathbf{1}_{N}=\left[\begin{array}{llll}1 & 1 & \ldots & 1\end{array}\right]^{\mathrm{T}} \in \mathbb{R}^{N \times 1}$ and the vector $\mathbf{L}=\left(v_{f}(2 / \pi) \arctan \left\{k_{\theta} e_{i}+k_{\dot{\theta}} \dot{e}_{i}\right\}\right)_{i=\overline{1, N}} \in \mathbb{R}^{N \times 1}$ should be found in the context of (1), $k_{\theta}$ is the positive tuning factor, $k_{\dot{\theta}}$ is the positive tuning factor for the derivative signal, $v_{f}$ is the maximum additional speed vector norm that must be set in the light of the constraints (3), $v$ is the final linear cruise speed of the UAV (in case of stationary target).

For UAV heading angle, the modified control law is written as:

$$
\chi^{c}=\left(\begin{array}{l}
\varphi_{i}+ \\
+\lambda\left[\frac{\pi}{2}+\arctan \left(k_{o}\left(d_{i}-\rho\right)+k_{\dot{o}} \dot{d}_{i}\right)\right]
\end{array}\right)_{i=\overline{1, N}} \in \mathbb{R}^{N \times 1},(6)
$$

where $d_{i}$ is the distance from the $i$ th UAV to the target, $k_{o}$ is the tuning factor for the distance-to-target signal, $k_{\dot{o}}$ is the tuning factor for the distance-to-target derivative signal; the rest of the notation is as in (4).

Thus, the control laws (5) and (6) set vector field for following a moving path in the UAV flight space; the field $F: \mathbb{R}^{2} \rightarrow \mathbb{R}^{2}$ is non-uniform in magnitude and direction alike. This field enables the system to generate appropriate heading angle and speed commands at any point in space. Thus, it enables the UAVs to attain and maintain a specified formation rotating around a moving target.

\section{Numerical SimUlation Results}

\section{A. Model in use and simulation parameters}

To verify the proposed control strategy computationally in MATLAB/Simulink, the authors hereof used complete nonlinear dynamics model of four flying-wing UAVs with 6 states and 12 degrees of freedom. Each UAV was also supplemented with an autopilot tuned by Successive Loop Closure [22].

Table I shows the simulation parameters. UAV specifications can be found in [22].

TABLE I. SIMULATION PARAMETERS

\begin{tabular}{|c|c|}
\hline Parameter & Value \\
\hline $\begin{array}{c}\text { Vector of initial UAV course } \\
\text { angles, [rad] }\end{array}$ & {$\left[\begin{array}{llll}0 & 0 & 0 & 0\end{array}\right]^{\mathrm{T}}$} \\
\hline $\begin{array}{l}\text { Vector of initial UAV speeds, } \\
{[\mathrm{m} / \mathrm{s}]}\end{array}$ & {$\left[\begin{array}{llll}13 & 13 & 13 & 13\end{array}\right]^{\mathrm{T}}$} \\
\hline $\begin{array}{l}\text { Ultimate cruise speed of the } \\
\text { formation, stationary target, }[\mathrm{m} / \mathrm{s}]\end{array}$ & 13 \\
\hline Target movement speed, $[\mathrm{m} / \mathrm{s}]$ & 2 \\
\hline Target course angle, $[\mathrm{rad}]$ & $\pi / 4$ \\
\hline $\begin{array}{c}\text { Initial coordinates of the 1st } \\
\text { UAV, ICS, [m] }\end{array}$ & {$\left[\begin{array}{lll}190 & 920 & 100\end{array}\right]^{\mathrm{T}}$} \\
\hline $\begin{array}{l}\text { Initial coordinates of the } 2 \mathrm{nd} \\
\text { UAV, ICS, [m] }\end{array}$ & {$\left[\begin{array}{lll}255 & 250 & 100\end{array}\right]^{\mathrm{T}}$} \\
\hline $\begin{array}{c}\text { Initial coordinates of the } 3 \mathrm{rd} \\
\text { UAV, ICS, [m] }\end{array}$ & {$\left[\begin{array}{lll}720 & 145 & 100\end{array}\right]^{\mathrm{T}}$} \\
\hline $\begin{array}{l}\text { Initial coordinates of the } 4 \text { th } \\
\text { UAV, ICS, [m] }\end{array}$ & {$\left[\begin{array}{lll}920 & 770 & 100\end{array}\right]^{\mathrm{T}}$} \\
\hline $\begin{array}{l}\text { Desired inter-UAV phase shift } \\
\text { angles, }\left[^{\circ}\right]\end{array}$ & {$\left[\begin{array}{lll}270 & 260 & 290\end{array}\right]^{\mathrm{T}}$} \\
\hline Tuning factors $k_{\theta}, k_{\dot{\theta}}, k_{o}, k_{\dot{o}}$ & $40,35,1,4$ \\
\hline
\end{tabular}

\begin{tabular}{|c|c|}
\hline Parameter & Value \\
\hline$\rho$, path circle radius, $[\mathrm{m}]$ & 200 \\
\hline $\begin{array}{c}\mathbf{c}=\left(c_{e}(0), c_{n}(0), h(0)\right), \text { initial } \\
\text { target coordinates, }[\mathrm{m}]\end{array}$ & {$\left[\begin{array}{lll}500 & 500 & 100\end{array}\right]$} \\
\hline
\end{tabular}

\section{B. Simulation Output}

Figures 1 to 4 show the output of simulation based on the control laws (5) and (6). Apparently, the UAVs are able to maintain the specified phase shift angles while following the specified moving path, both with a fair degree of accuracy. This effectively solves two problem: shaping a formation and standoff tracking.

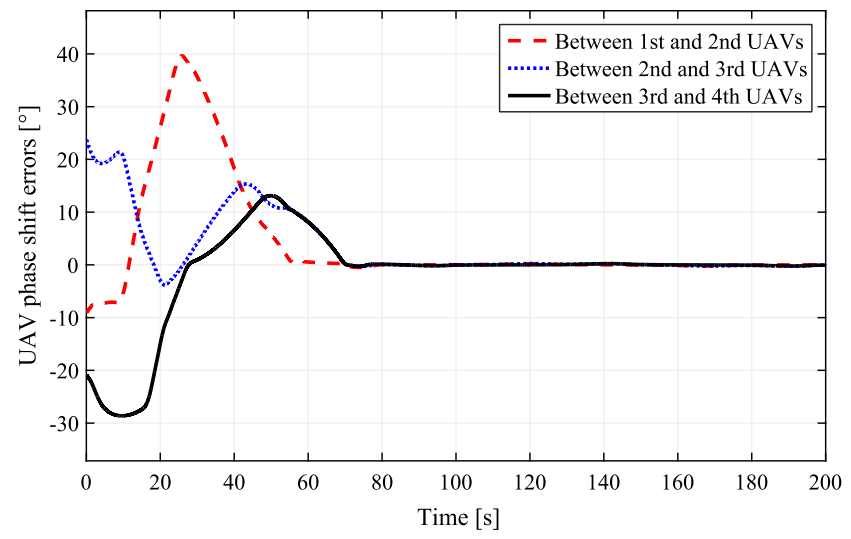

Fig. 1. Changes in inter-UAV phase shift errors over time

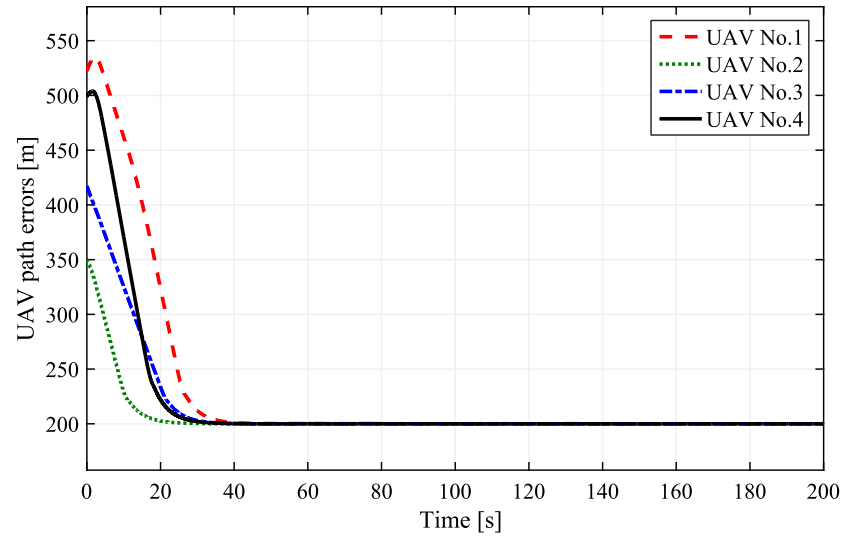

Fig. 2. Changes in UAV path errors over time 


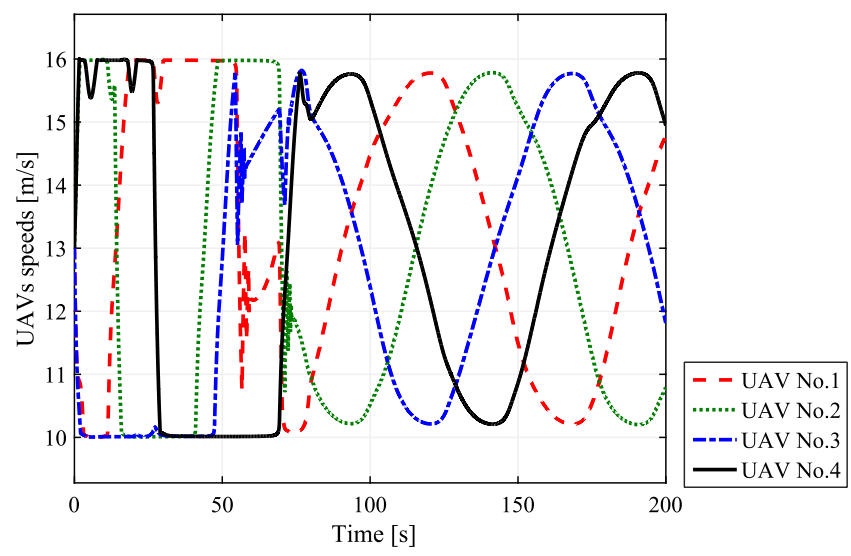

Fig. 3. Changes in UAV speeds over time

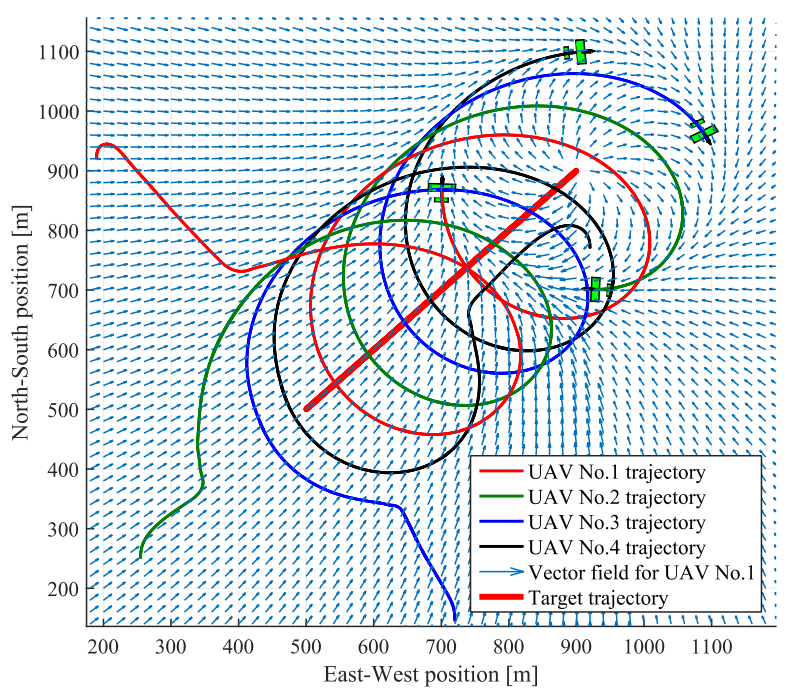

Fig. 4. Trajectories of four UAVs involved in cooperative target tracking, target trajectory, and path following vector field for a single UAV at the time $t=200 \mathrm{~s}$

Figures 1 to 4 apply to UAVs flying in a formation. For comparison, Figures 5 and 6 show how phase shift and path errors would change by not using derivative signals in control laws. Simulation clearly shows neither formation attainment nor path following would be stable.

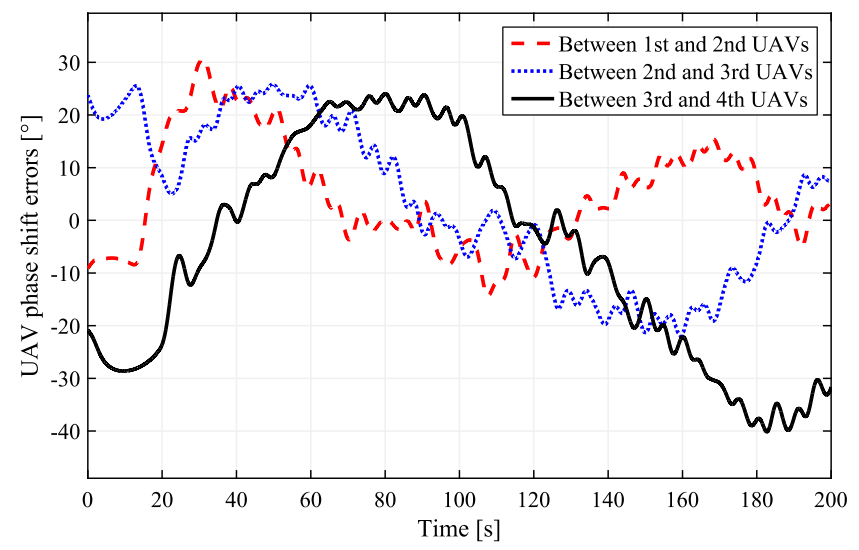

Fig. 5. Changes in inter-UAV phase shift errors over time, no derivative signals

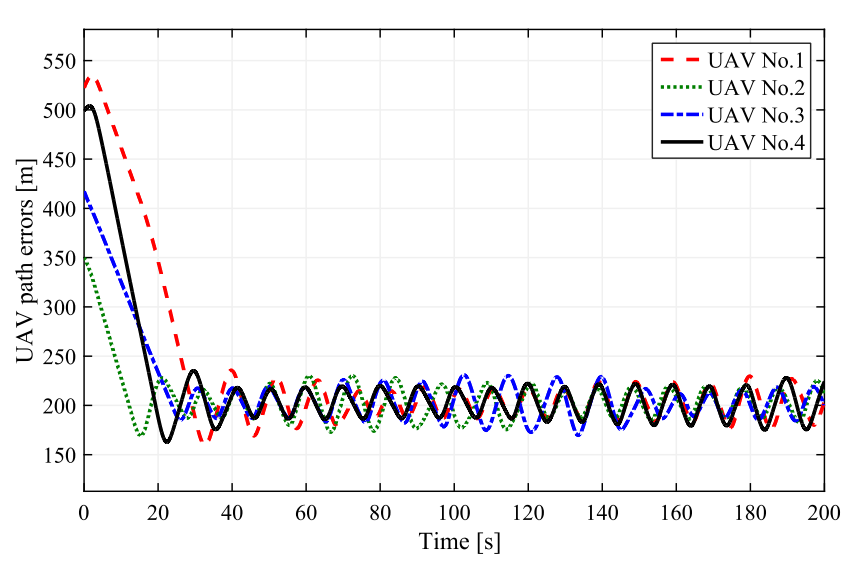

Fig. 6. Changes in UAV path errors over time, no derivative signals

\section{CONCLUSION}

This paper describes an approach to cooperative tracking of ground targets by fixed-wing UAVs. To fly while keeping a set distance to the target and set inter-UAV spacings, this paper proposes using a novel guidance vector field that is non-uniform in both the direction and the magnitude. Thanks to this, the vector field enables the formation to follow a moving circular path while also attaining any specified phase shift angle. At the same time, complete decentralization of coordination means the group can comprise any number of UAVs.

Numerical simulation by complete nonlinear models demonstrates this strategy feasible. For space considerations, this paper only describes targets following a rectilinear trajectory at a constant speed; thus, further research will cover more complex trajectories and how the UAVs themselves could estimate it.

\section{REFERENCES}

[1] V. I. Goncharenko, G. N. Lebedev, and D. A. Mikhailin, "Online TwoDimensional Route Planning for a Group of Unmanned Aerial Vehicles," J. Comput. Syst. Sci. Int., vol. 58, no. 1, pp. 147-158, Jan. 2019.

[2] V. Pshikhopov, M. Medvedev, and T. Medvedeva, "Terminal Motion Control of Multicopter Group," in 4th International Conference on Control and Robotics Engineering, ICCRE 2019, 2019, pp. 1-6.

[3] H. Qiu and H. Duan, "Pigeon interaction mode switch-based UAV distributed flocking control under obstacle environments," ISA Trans., vol. 71, pp. 93-102, Nov. 2017.

[4] S. Park and D. Jung, "Vision-Based Tracking of a Ground-Moving Target with UAV," Int. J. Aeronaut. Sp. Sci., vol. 20, no. 2, pp. 467-482, Jun. 2019.

[5] E. W. Frew, "Sensitivity of cooperative target geolocalization to orbit coordination," J. Guid. Control. Dyn., vol. 31, no. 4, pp. 1028-1040, Jul. 2008.

[6] D. Lawrence, E. Frew, and W. Pisano, "Lyapunov vector fields for autonomous UAV flight control," AIAA Guid. Navig. Control Conf. Exhib., p. 6317, 2007.

[7] E. W. Frew, D. A. Lawrence, and S. Morris, "Coordinated standoff tracking of moving targets using Lyapunov guidance vector fields," $J$. Guid. Control. Dyn., vol. 31, no. 2, pp. 290-306, 2008.

[8] T. H. Summers, M. R. Akella, and M. J. Mears, "Coordinated standoff tracking of moving targets: Control laws and information architectures," J. Guid. Control. Dyn., vol. 32, no. 1, pp. 56-69, 2009. 
[9] H. Oh, S. Kim, A. Tsourdos, and B. A. White, "Decentralised standoff tracking of moving targets using adaptive sliding mode control for UAVs," J. Intell. Robot. Syst., vol. 76, no. 1, pp. 169-183, 2014.

[10] D. Kingston and R. W. Beard, "UAV splay state configuration for moving targets in wind," in Advances in Cooperative Control and Optimization, Berlin: Springer, 2007, pp. 109-128.

[11] L. Ma, "Cooperative Target Tracking by Altering UAVs' Linear and Angular Velocities," in 2019 International Conference on Unmanned Aircraft Systems (ICUAS), 2019, pp. 69-78.

[12] L. Briñón-Arranz, A. Seuret, and A. Pascoal, "Circular formation control for cooperative target tracking with limited information," J. Franklin Inst., vol. 356, no. 4, pp. 1771-1788, Mar. 2019.

[13] T. Z. Muslimov and R. A. Munasypov, "Consensus-Based Cooperative Circular Formation Control Strategy for Multi-UAV System," in International Russian Automation Conference (RusAutoCon), 2019, pp. $1-8$.

[14] Y. A. Kapitanyuk, H. G. de Marina, A. V. Proskurnikov, and M. Cao, "Guiding vector field algorithm for a moving path following problem," IFAC-PapersOnLine, vol. 50, no. 1, pp. 6983-6988, Jul. 2017.

[15] T. Oliveira, A. P. Aguiar, and P. Encarnação, "Moving Path Following for Unmanned Aerial Vehicles With Applications to Single and Multiple Target Tracking Problems," IEEE Trans. Robot., vol. 32, no. 5, pp. 1062-1078, Oct. 2016.
[16] H.-S. Ahn, "Preliminary Background," in Formation Control. Studies in Systems, Decision and Control, Cham: Springer, 2020, pp. 3-26.

[17] T. Z. Muslimov and R. A. Munasypov, "Decentralized Nonlinear Group Control of Fixed-wing UAV Formation," Mekhatronika, Avtom. Upr., vol. 21, no. 1, pp. 43-50 (in Russian), 2020.

[18] T. Z. Muslimov, "Algorithms of Autonomous Fixed-wing UAVs Formation Control via Vector Field Method," Syst. Control. Commun. Secur., no. 4, pp. 187-214 (in Russian), 2019.

[19] H. Yamaguchi, T. Arai, and G. Beni, "A distributed control scheme for multiple robotic vehicles to make group formations," Rob. Auton. Syst., vol. 36, no. 4, pp. 125-147, Sep. 2001.

[20] H. Yamaguchi and T. Arai, "Distributed and autonomous control method for generating shape of multiple mobile robot group," Proc. IEEE/RSJ Int. Conf. Intell. Robot. Syst., vol. 2, pp. 800-807, 1994.

[21] R. W. Beard, J. Ferrin, and J. Humpherys, "Fixed wing UAV path following in wind with input constraints," IEEE Trans. Control Syst. Technol., vol. 22, no. 6, pp. 2103-2117, 2014.

[22] R. W. Beard and T. W. McLain, Small unmanned aircraft: Theory and practice. Princeton University Press, 2012. 